\title{
On a Differential Inclusion Involving Dirichlet-Laplace Operators of Fractional Orders
}

\author{
Rafał Kamocki ${ }^{1}$ \\ Received: 2 July 2019 / Revised: 24 November 2019 / Published online: 18 February 2020 \\ (c) The Author(s) 2020
}

\begin{abstract}
In this paper, we investigate a nonlinear differential inclusion with Dirichlet boundary conditions containing a weak Laplace operator of fractional orders (defined via the spectral decomposition of the Laplace operator $-\Delta$ under Dirichlet boundary conditions). Using variational methods, we characterize solutions of such a problem. Our approach is based on tools from convex analysis (properties of a Legendre-Fenchel transform).
\end{abstract}

Keywords Fractional Dirichlet-Laplace operator · Differential inclusion ·

Legendre-Fenchel transform · Variational methods

Mathematics Subject Classification 35R20 - 47F05 · 47B25

\section{Introduction}

In recent years, a fractional Laplacian (more precisely, the fractional powers of the Laplacian) attracted attention of many scientists due to its applications in various areas. It appears including in probability (cf. [1,6,7,13]), economics and finance (cf. $[1,14]$ ), mechanics (cf. [5,7]), optimal control theory (cf. [9,20]), fluid mechanics and hydrodynamics (cf. [8,10-12,28-30]). There exist many definitions of such operators (e.g., Fourier transform [15,17], hypersingular integral [15], Riesz potential operator [24], Bochner's definition [27], spectral decomposition (cf. [4,14,18]).

\section{Communicated by Rosihan M. Ali.}

Rafał Kamocki

rafal.kamocki@wmii.uni.lodz.pl

1 Faculty of Mathematics and Computer Science, University of Lodz, Banacha 22, 90-238 Lodz, Poland 
In this paper, we are concerned with the study of solutions to the following differential inclusion

$$
\sum_{i=0}^{k} \alpha_{i}\left[(-\Delta)_{\omega}\right]^{\beta_{i}} u(x) \in \partial_{u} F(x, u(x)), \quad x \in \Omega \text { a.e. }
$$

where $\alpha_{i}>0, i=0, \ldots, k(k \in \mathbb{N} \cup\{0\}), 0 \leq \beta_{0}<\beta_{1}<\cdots<\beta_{k}, \Omega \subset \mathbb{R}^{N}$ is an open and bounded set, $F: \Omega \times \mathbb{R} \rightarrow[0, \infty), \partial_{u} F$ denotes a subgradient of $F$ with respect to $u,\left[\left(-\Delta_{\omega}\right)\right]^{\gamma}$ denotes a weak fractional Laplace operator of order $\gamma>0$ with zero Dirichlet boundary values on $\partial \Omega$ (the term "weak" is explained in Sect. 2). This work is based on the spectral definition of the mentioned operator (we shall call it a weak fractional Dirichlet-Laplace operator). More precisely, the definition of the Dirichlet Laplacian comes from the functional calculus for unbounded self-adjoint operators in a Hilbert space $[16,21]$ and is based on the spectral integral representation theorem for such operators $[23,25]$.

Let us note that if $F(t, \cdot)$ is differentiable on $\mathbb{R}$, then (1) reduces to the following boundary value problem

$$
\sum_{i=0}^{k} \alpha_{i}\left[(-\Delta)_{\omega}\right]^{\beta_{i}} u(x)=D_{u} F(x, u(x)), \quad x \in \Omega \text { a.e. }
$$

Motivated by the paper [3], we characterize solutions of the problem (1). They are minimizers of a some integral functional $J$ related to (1) due to the Legendre-Fenchel transform of the function $F$ and the Fenchel-Young inequality (cf. Theorem 3). Using a standard method of the calculus of variations, we prove the existence and uniqueness of minimizers of $J$ on an appropriate space of functions, being the space of solutions of (1) (cf. Theorem 4). Finally, obtained results are illustrated by some examples. In particular, we show that if $F$ is adequately chosen, then the minimizer of $J$ is a solution of (2). To the best of our knowledge, the problem of a type (1) was not investigated by other authors.

\section{Preliminaries}

In this section, we give some preliminary notions and results that will be used in the remaining part of this paper.

\subsection{Weak Fractional Dirichlet-Laplace Operator}

In a whole paper we assume that $\Omega \subset \mathbb{R}^{N}$ is an open and bounded set.

Let $T_{0}=-\Delta: C_{c}^{\infty}(\Omega, \mathbb{R}) \subset L^{2}(\Omega, \mathbb{R}) \rightarrow L^{2}(\Omega, \mathbb{R})$ be the classical DirichletLaplace operator. 
Definition 1 ( $c f$. [21]) We say that $u: \Omega \rightarrow \mathbb{R}$ has a weak (minus) Dirichlet-Laplacian if $u \in H_{0}^{1}(\Omega, \mathbb{R})$ and there exists a function $g \in L^{2}(\Omega, \mathbb{R})$ such that

$$
\int_{\Omega} \nabla u(x) \nabla v(x) \mathrm{d} x=\int_{\Omega} g(x) v(x) \mathrm{d} x
$$

for any $v \in H_{0}^{1}(\Omega, \mathbb{R})$. The function $g$ will be called the weak Dirichlet-Laplacian and denoted by $(-\Delta)_{\omega} u$.

The operator $(-\Delta)_{\omega}$ is called the weak Dirichlet-Laplace operator and the set

$$
\begin{aligned}
& D\left((-\Delta)_{\omega}\right) \\
& \quad:=\left\{u \in H_{0}^{1}(\Omega, \mathbb{R}) ; \quad \exists_{g \in L^{2}}, \quad \forall_{v \in H_{0}^{1}} \int_{\Omega} \nabla u(x) \nabla v(x) \mathrm{d} x=\int_{\Omega} g(x) v(x) \mathrm{d} x\right\}
\end{aligned}
$$

is named the domain of the operator $(-\Delta)_{\omega}$.

Using the Friedrich's extension procedure, we obtain (cf. [21, Theorem 3.1], [23])

Theorem 1 The operator

$$
(-\Delta)_{\omega}: D\left((-\Delta)_{\omega}\right) \subset L^{2}(\Omega, \mathbb{R}) \rightarrow L^{2}(\Omega, \mathbb{R})
$$

is the self-adjoint extension of the operator $T_{0}$ and

$$
T_{0} \subset-\Delta \subset(-\Delta)_{\omega}
$$

where $-\Delta: H_{0}^{1}(\Omega, \mathbb{R}) \cap H^{2}(\Omega, \mathbb{R}) \subset L^{2}(\Omega, \mathbb{R}) \rightarrow L^{2}(\Omega, \mathbb{R})$ is the (strong) Dirichlet-Laplace operator.

Remark 1 In [2, Section 8.2] $(-\Delta)_{\omega}$ is called the Laplace-Dirichlet operator (without "weak") and denoted by $-\Delta$.

It is known (cf. [21]) that the spectrum $\sigma\left((-\Delta)_{\omega}\right)$ of $(-\Delta)_{\omega}$ contains only the eigenvalues of $(-\Delta)_{\omega}$ that can be written in a non-decreasing sequence, repeating each eigenvalue according to its multiplicity $0<\lambda_{1} \leq \lambda_{2} \leq \cdots \leq \lambda_{j} \rightarrow \infty$. Moreover, a system $\left\{e_{j}\right\}$ of eigenfunctions of the operator $(-\Delta)_{\omega}$, corresponding to $\lambda_{j}$, is a hilbertian basis in $L^{2}(\Omega, \mathbb{R})$.

Remark 2 In paper [21], the author proved that if $\Omega$ is an open and bounded set of class $C^{1,1}$ or a convex polygon in $\mathbb{R}^{2}$, then the weak and strong Dirichlet-Laplace operators coincide.

Now, let $\beta>0$. By the weak fractional Dirichlet-Laplace operator of order $\beta$ we mean the operator $\left[(-\Delta)_{\omega}\right]^{\beta}: D\left(\left[(-\Delta)_{\omega}\right]^{\beta}\right) \subset L^{2}(\Omega, \mathbb{R}) \rightarrow L^{2}(\Omega, \mathbb{R})$ defined in the following way (cf. [21, Section 3]): 


$$
\left(\left[(-\Delta)_{\omega}\right]^{\beta} u\right)(t)=\left(\left(\int_{\sigma\left((-\Delta)_{\omega}\right)} \lambda^{\beta} E(\mathrm{~d} \lambda)\right) u\right)(t)=\sum_{j=1}^{\infty}\left(\lambda_{j}\right)^{\beta} a_{j} e_{j}(t)
$$

where

$$
\begin{aligned}
D\left(\left[(-\Delta)_{\omega}\right]^{\beta}\right)= & \left\{u \in L^{2}(\Omega, \mathbb{R}) ; \int_{\sigma\left((-\Delta)_{\omega}\right)}\left|\lambda^{\beta}\right|^{2}\|E(\mathrm{~d} \lambda) u\|^{2}=\sum_{j=1}^{\infty}\left(\left(\lambda_{j}\right)^{\beta}\right)^{2} a_{j}^{2}<\infty,\right. \\
& \text { where } a_{j} \text {-s is such that } u(t)=\left(\left(\int_{\sigma\left((-\Delta)_{\omega}\right)} 1 E(\mathrm{~d} \lambda)\right) u\right)(t) \\
= & \left.\sum_{j=1}^{\infty} a_{j} e_{j}(t)\right\}
\end{aligned}
$$

(here $E$ is the spectral measure given by $(-\Delta)_{\omega}$ and the convergence of the series is meant in $\left.L^{2}(\Omega, \mathbb{R})\right)$.

It is well known that the operator $\left[(-\Delta)_{\omega}\right]^{\beta}$ is self-adjoint, its spectrum $\sigma\left(\left[(-\Delta)_{\omega}\right]^{\beta}\right)$ contains only proper values $\left(\lambda_{j}\right)^{\beta}, j \in \mathbb{N}$. Moreover, eigenspaces, corresponding to $\left(\lambda_{j}\right)^{\beta}$-s and eigenspaces for $\left[(-\Delta)_{\omega}\right]$, corresponding to $\lambda_{j}$-s are the same.

In the space $D\left(\left[(-\Delta)_{\omega}\right]^{\beta}\right)$, we define two scalar products:

$$
\begin{aligned}
\langle u, v\rangle_{\beta} & :=\langle u, v\rangle_{L^{2}}+\left\langle\left[(-\Delta)_{\omega}\right]^{\beta} u,\left[(-\Delta)_{\omega}\right]^{\beta} v\right\rangle_{L^{2}}, \\
\langle u, v\rangle_{\sim \beta} & :=\left\langle\left[(-\Delta)_{\omega}\right]^{\beta} u,\left[(-\Delta)_{\omega}\right]^{\beta} v\right\rangle_{L^{2}}
\end{aligned}
$$

which generate norms:

$$
\begin{aligned}
\|u\|_{\beta} & =\left(\|u\|_{L^{2}}^{2}+\left\|\left[(-\Delta)_{\omega}\right]^{\beta} u\right\|_{L^{2}}^{2}\right)^{\frac{1}{2}}, \\
\|u\|_{\sim \beta} & =\left\|\left[(-\Delta)_{\omega}\right]^{\beta} u\right\|_{L^{2}},
\end{aligned}
$$

respectively. Norms (3) and (4) are equivalent due to the following Poincaré inequality in $D\left(\left[(-\Delta)_{\omega}\right]^{\beta}\right)$ (cf. [21, inequality (3.2)]):

$$
\|u\|_{L^{2}}^{2} \leq M_{\beta}\|u\|_{\sim \beta}^{2},
$$

where

$$
M_{\beta}= \begin{cases}1 & \text { if } \lambda_{1} \geq 1 \\ \frac{1}{\left(\left(\lambda_{1}\right)^{\beta}\right)^{2}} & \text { if } \quad \lambda_{1}<1\end{cases}
$$

(here $\lambda_{1}>0$ is the first (the smallest) eigenvalue of the operator $(-\Delta)_{\omega}$ ). 
Now, let us consider the function

$$
w: \mathbb{R} \ni \lambda \rightarrow \begin{cases}0 & \lambda<0 \\ \alpha_{k} \lambda^{\beta_{k}}+\cdots+\alpha_{1} \lambda^{\beta_{1}}+\alpha_{0} \lambda^{\beta_{0}} & \lambda \geq 0,\end{cases}
$$

where $0 \leq \beta_{0}<\beta_{1}<\cdots<\beta_{k}, k \in \mathbb{N} \cup\{0\}, \alpha_{i}>0, i=0, \ldots, k$. Then, we can define the operator $w\left((-\Delta)_{\omega}\right): D\left(w\left((-\Delta)_{\omega}\right)\right) \subset L^{2}(\Omega, \mathbb{R}) \rightarrow L^{2}(\Omega, \mathbb{R})$ as follows:

$$
w\left((-\Delta)_{\omega}\right) u:=\sum_{i=0}^{k} \alpha_{i}\left((-\Delta)_{\omega}\right)^{\beta_{i}} u .
$$

The operator $w\left((-\Delta)_{\omega}\right)$ is self-adjoint and $D\left(w\left((-\Delta)_{\omega}\right)\right)=D\left(\left((-\Delta)_{\omega}\right)^{\beta_{k}}\right)$ (cf. [21]). Moreover, in $D\left(\left[(-\Delta)_{\omega}\right]^{\beta_{k}}\right)$ one can introduce a new scalar product of the form

$$
\langle u, v\rangle_{w\left((-\Delta)_{\omega}\right)}:=\left\langle w\left((-\Delta)_{\omega}\right) u, w\left((-\Delta)_{\omega}\right) v\right\rangle_{L^{2}}
$$

which generates a norm

$$
\|u\|_{w\left((-\Delta)_{\omega}\right)}=\left\|w\left((-\Delta)_{\omega}\right) u\right\|_{L^{2}} .
$$

Norms (4) and (8) (the norm (8) will be used in the main part of this article) are equivalent in $D\left(\left[(-\Delta)_{\omega}\right]^{\beta_{k}}\right)$ (cf. [21, Lemma 3.6]) and

$$
\|u\|_{\sim \beta_{k}}^{2} \leq \frac{1}{\alpha_{k}^{2}}\|u\|_{w\left((-\Delta)_{\omega}\right)}^{2} \leq \frac{C_{1}}{\alpha_{k}^{2}}\left(1+k C_{2}^{2}\right)\|u\|_{\sim \beta_{k}}^{2},
$$

where $C_{1}, C_{2}>0$ are constants from [21, Lemma 3.6]. Hence and from (5) we obtain the following Poincaré inequality in $D\left(\left[(-\Delta)_{\omega}\right]^{\beta_{k}}\right)$ :

$$
\|u\|_{L^{2}}^{2} \leq \frac{1}{M_{\beta} \alpha_{k}^{2}}\|u\|_{w\left((-\Delta)_{\omega}\right)}^{2}
$$

Moreover, the space $D\left(w\left((-\Delta)_{\omega}\right)\right)=D\left(\left((-\Delta)_{\omega}\right)^{\beta_{k}}\right)$ with the scalar product $(7)$ is complete (cf. [21, Lemma 3.6]).

\subsection{Basic Facts from Convex Analysis}

In this part we recall some basic definitions and facts concerning the convex analysis. More details can be found in [19].

Let $f: \mathbb{R}^{n} \rightarrow \mathbb{R}$. We shall say that a vector $y \in \mathbb{R}^{n}$ is a subgradient of $f$ at $x \in \mathbb{R}^{n}$ if

$$
f(z)-f(x) \geqslant\langle y, z-x\rangle_{\mathbb{R}^{n}}, \quad \text { for every } z \in \mathbb{R}^{n} .
$$


The set of all subgradients of $f$ at $x$ is called a subdifferential and is denoted by $\partial f(x)$. If $\partial f(x) \neq \emptyset$ then the function $f$ is called subdifferentiable at $x$. If $f$ is a convex function, then the subdifferential is a nonempty, convex and compact set. Moreover, if $f$ is Gateaux differentiable at $x$, then $\partial f(x)=\{\nabla f(x)\}$.

The function $f^{*}: \mathbb{R}^{n} \rightarrow \mathbb{R} \cup\{\infty\}$ defined by

$$
f^{*}(y):=\sup _{x \in \mathbb{R}^{n}}\left\{\langle y, x\rangle_{\mathbb{R}^{n}}-f(x)\right\}
$$

is called the Legendre-Fenchel transform of the function $f$.

In the main part of this paper we use the following facts:

$$
f(x) \leq g(x), x \in \mathbb{R}^{n} \Longrightarrow f^{*}(y) \geq g^{*}(y), \quad y \in \mathbb{R}^{n} .
$$

Theorem 2 (Fenchel-Young inequality) Let $f: \mathbb{R}^{n} \rightarrow \mathbb{R}$. Any points $x, y \in \mathbb{R}^{n}$ satisfy the inequality

$$
f(x)+f^{*}(y) \geqslant\langle y, x\rangle \text {. }
$$

Equality holds if and only if $y \in \partial f(x)$.

In conclusion of this section we give an another useful fact (cf. [22, Part II, Lemma 4.3.1]).

Lemma 1 Let $\Omega \subset \mathbb{R}^{d}$ be open, $f: \Omega \times \mathbb{R}^{d} \rightarrow \mathbb{R}$, with $f(\cdot, v)$ measurable for all $v \in \mathbb{R}^{d}, f(x, \cdot)$ continuous for almost all $x \in \Omega$, and

$$
f(x, v) \geq-a(x)+b|v|^{p}, \quad x \in \Omega \text { a.e., } v \in \mathbb{R}^{d},
$$

where $a \in L^{1}(\Omega, \mathbb{R}), b \in \mathbb{R}, p \geq 1$. Then, the functional $\Phi: L^{p}\left(\Omega, \mathbb{R}^{d}\right) \rightarrow \mathbb{R} \cup\{\infty\}$,

$$
\Phi(v):=\int_{\Omega} f(x, v(x)) \mathrm{d} x
$$

is a sequentially lower semicontinuous on $L^{p}\left(\Omega, \mathbb{R}^{d}\right)$.

Remark 3 The above lemma can be also proved in case of the function $f$ defined on $\Omega \times \mathbb{R}^{n}$, where $n \neq d$.

\section{Differential Inclusion with Fractional Dirichlet-Laplace Operators}

In this section we prove the main result of this paper, namely a theorem on the existence of minimizers of a some functional associated with the problem (1).

By a solution of inclusion (1) we mean a function $u \in D\left(w\left((-\Delta)_{\omega}\right)\right)$ satisfying (1) a.e. on $\Omega$. 
Let us assume that $F$ satisfies the following conditions:

(F1): $F(\cdot, u)$ is measurable on $\Omega$ for all $u \in \mathbb{R}$ and $F(x, \cdot)$ is convex on $\mathbb{R}$ for a.e. $x \in \Omega$.

(F2): $F(x, \cdot)$ is coercive on $\mathbb{R}$ for a.e. $x \in \Omega$, i.e.

$$
\lim _{|u| \rightarrow \infty} \frac{F(x, u)}{|u|}=\infty, \quad x \in \Omega \text { a.e. }
$$

(F3): there exist a function $v \in L^{1}(\Omega, \mathbb{R})$ and a constant $c>0$ such that

$$
F(x, u) \leq v(x)+c|u|^{2}, \quad x \in \Omega \text { a.e., } u \in \mathbb{R} .
$$

Remark 4 Of course, since $F(x, \cdot)$ is convex, therefore it is continuous on $\mathbb{R}$ for a.e. $x \in \Omega$ (cf. [26, Corollary 10.1.1]).

Let us consider the function $f: \mathbb{R} \rightarrow \mathbb{R}$ given by

$$
f(u):=v+c|u|^{2}
$$

where $c>0$ and $v \in \mathbb{R}$ are fixed. Then, it easy to check that

$$
f^{*}(z)=-v+\frac{1}{4 c}|z|^{2}, \quad z \in \mathbb{R} .
$$

In the next result we shall use the following

Lemma 2 Let $G: \Omega \times \mathbb{R} \rightarrow \mathbb{R}$ be a Carathéodory function and the function $H$ : $\Omega \rightarrow \mathbb{R} \cup\{\infty\}$ is defined by

$$
H(x):=\sup _{w \in \mathbb{R}}\{G(x, w)\}, \quad x \in \Omega \text { a.e. }
$$

Then $H$ is measurable on $\Omega$.

Proof From the fact that $G(\cdot, w)$ is continuous on $\mathbb{R}$ it follows that

$$
H(x)=\sup _{w \in \mathbb{Q}}\{G(x, w)\}, \quad x \in \Omega \text { a.e. }
$$

where $\mathbb{Q}$ denotes the set of rational numbers. Since the supremum of the countable set of measurable functions is a measurable set, therefore $H$ is measurable on $\Omega$.

Now, we formulate and prove the following result.

Proposition 1 Let us assume that conditions (F1)-(F3) are satisfied. Then, the Legendre-Fenchel transform of the function $F$ with respect to $u F^{*}: \Omega \times \mathbb{R} \rightarrow$ $(-\infty, \infty]$ given by

$$
F^{*}(x, z):=\sup _{u \in \mathbb{R}}\left[\langle u, z\rangle_{\mathbb{R}}-F(x, u)\right], \quad x \in \Omega \text { a.e., } z \in \mathbb{R}
$$


has the following properties:

(a) $F^{*}(x, z)$ is finite for a.e. $x \in \Omega$ and all $z \in \mathbb{R}$,

(b) $F^{*}(\cdot, z)$ is measurable on $\Omega$ for all $z \in \mathbb{R}$ and $F^{*}(x, \cdot)$ is continuous on $\mathbb{R}$ for a.e. $x \in \Omega$,

(c) for a.e. $x \in \Omega$ and all $z \in \mathbb{R}$

$$
F^{*}(x, z) \geq-v(x)+\frac{1}{4 c}|z|^{2}
$$

where the constant $c$ and the function $v$ are from assumption $(F 3)$.

Proof proof of the property (a): Let us fix any $z \in \mathbb{R}$ and $x \in S \subset \Omega$, where $\mu(\Omega \backslash S)=0$ ( $\mu$ denotes the Lebesque measure on $\left.\mathbb{R}^{N}\right)$. From (F2) it follows that for any $R>0$ there exists $r>0$ such that for $|u|>r$ we have $F(x, u) \geq R|u|$. In particular, putting $R=|z|$, we get

$$
F(x, u) \geq|z||u|, \quad|u|>r .
$$

Consequently,

$$
\langle u, z\rangle_{\mathbb{R}}-F(x, u) \leq|u||z|-R|u|=0, \quad|u|>r .
$$

Hence and from continuity of the function $F(\cdot, u)$ we obtain

$$
\begin{aligned}
F^{*}(x, z) & =\sup _{u \in \mathbb{R}}\left[\langle u, z\rangle_{\mathbb{R}}-F(x, u)\right] \\
& =\max \left\{\sup _{|u| \leq r}\left[\langle u, z\rangle_{\mathbb{R}}-F(x, u)\right], \sup _{|u|>r}\left[\langle u, z\rangle_{\mathbb{R}}-F(x, u)\right]\right\} \\
& \leq \max \{\text { const, } 0\}<\infty .
\end{aligned}
$$

This means that $F^{*}$ is finite for a.e. $x \in \Omega$ and all $z \in \mathbb{R}$.

proof of the property $(b)$ : Measurability of $F^{*}(\cdot, z)$ on $\Omega$ for any fixed $z \in \mathbb{R}$ follows from Lemma 2. From the definition of the Legendre-Fenchel transform it follows that $F^{*}(x, \cdot)$ is convex on $\mathbb{R}$ for a.e. $x \in \Omega$. Thus and from the proved condition $(a)$ of this proposition we obtain continuity of $F^{*}$ with respect to $z$ for a.e. $x \in \Omega$ (cf. [26, Corollary 10.1.1]).

proof of the property $(c)$ : The condition (16) follows from assumption (F3), property (11) and equality (15).

Now, let us consider the functional $J: D\left(w\left((-\Delta)_{\omega}\right)\right) \longrightarrow \mathbb{R} \cup\{\infty\}$ given by:

$$
J(u):=\int_{\Omega}\left(F(x, u(x))+F^{*}\left(x, w\left((-\Delta)_{\omega}\right) u(x)\right)-\left\langle u(x), w\left((-\Delta)_{\omega}\right) u(x)\right\rangle_{\mathbb{R}}\right) \mathrm{d} x .
$$


Let us note that since all terms in the above integral are measurable on $\Omega$ (the first and third terms are even summable on $\Omega$ ), therefore $J$ is well defined.

In the proof of the main result we use the following fact.

Lemma 3 If assumptions (F1)-(F3) are satisfied, then the functional $I: D\left(w\left((-\Delta)_{\omega}\right)\right) \rightarrow \mathbb{R} \cup\{\infty\}$ given by

$$
\left.I(u)=\int_{\Omega} F^{*}\left(x, w\left((-\Delta)_{\omega}\right) u(x)\right)\right) \mathrm{d} x
$$

is sequentially weakly lower semicontinuous on $D\left(w\left((-\Delta)_{\omega}\right)\right)$.

Proof Let us consider the functional $I_{1}: L^{2}(\Omega, \mathbb{R}) \rightarrow \mathbb{R} \cup\{\infty\}$ given by

$$
I_{1}(z)=\int_{\Omega} F^{*}(x, z(x)) \mathrm{d} x .
$$

From Proposition 1, Lemma 1 and Remark 3 it follows that $I_{1}$ is a sequentially lower semicontinuous functional on $L^{2}(\Omega, \mathbb{R})$.

Let $\left(u_{n}\right)_{n \in \mathbb{N}} \subset D\left(w\left((-\Delta)_{\omega}\right)\right)$ be a sequence convergent to a some element $u$ in the space $D\left(w\left((-\Delta)_{\omega}\right)\right)$. Then, the sequence $\left(w((-\Delta) \omega) u_{n}\right)_{n \in \mathbb{N}} \subset L^{2}(\Omega, \mathbb{R})$ is convergent to $w((-\Delta) \omega) u$ in $L^{2}(\Omega, \mathbb{R})$. From the proved part of this Lemma it follows that the functional $I$ is sequentially lower semicontinuous on $D\left(w\left((-\Delta)_{\omega}\right)\right)$. Since $I$ is convex on $D\left(w\left((-\Delta)_{\omega}\right)\right)\left(F^{*}(x, \cdot)\right.$ is convex on $\left.\mathbb{R}\right)$, therefore it is sequential weak lower semicontinuous on $D\left(w\left((-\Delta)_{\omega}\right)\right)$.

The proof is completed.

The next theorem plays a key role in the study of the existence of solutions to problem (1).

Theorem 3 Assume that conditions (F1)-(F3) are satisfied. A function $u_{*} \in$ $D\left(w\left((-\Delta)_{\omega}\right)\right)$ is a solution of the inclusion (1) if and only if $u_{*}$ is a minimizer of $J$ on $D\left(w\left((-\Delta)_{\omega}\right)\right)$ and $J\left(u_{*}\right)=0$.

Proof Let $u_{*} \in D\left(w\left((-\Delta)_{\omega}\right)\right)$ be a solution of the problem (1). From Theorem 2 it follows that

$$
\begin{aligned}
& F\left(x, u_{*}(x)\right)+F^{*}\left(x, w\left((-\Delta)_{\omega}\right) u_{*}(x)\right)-\left\langle u_{*}(x), w\left((-\Delta)_{\omega}\right) u_{*}(x)\right\rangle_{\mathbb{R}}=0, \\
& \quad x \in \Omega \text { a.e. }
\end{aligned}
$$

Consequently, $J\left(u_{*}\right)=0$. On the other hand, for every $u \in D\left(w\left((-\Delta)_{\omega}\right)\right)$, using once again Theorem 2, we have

$$
F(x, u(x))+F^{*}\left(x, w\left((-\Delta)_{\omega}\right) u(x)\right)-\left\langle u(x), w\left((-\Delta)_{\omega}\right) u(x)\right\rangle_{\mathbb{R}} \geq 0, \quad x \in \Omega \text { a.e. }
$$


This means that $J(u) \geq 0$ for every $u \in D\left(w\left((-\Delta)_{\omega}\right)\right)$, so $u_{*}$ is a minimizer of $J$ on $D\left(w\left((-\Delta)_{\omega}\right)\right)$.

Now, we assume that $u_{*}$ is a minimizer of $J$ on $D\left(w\left((-\Delta)_{\omega}\right)\right)$ and $J\left(u_{*}\right)=0$. This means in particular that

$$
\begin{aligned}
0 & =J\left(u_{*}\right) \\
& =\int_{\Omega}\left(F\left(x, u_{*}(x)\right)+F^{*}\left(x, w\left((-\Delta)_{\omega}\right) u_{*}(x)\right)-\left\langle u_{*}(x), w\left((-\Delta)_{\omega}\right) u_{*}(x)\right\rangle_{\mathbb{R}}\right) \mathrm{d} x .
\end{aligned}
$$

On the other hand, from Theorem 2 it follows that

$$
\begin{aligned}
& F\left(x, u_{*}(x)\right)+F^{*}\left(x, w\left((-\Delta)_{\omega}\right) u_{*}(x)\right)-\left\langle u_{*}(x), w\left((-\Delta)_{\omega}\right) u_{*}(x)\right\rangle_{\mathbb{R}} \geq 0, \\
& \quad x \in \Omega \text { a.e. }
\end{aligned}
$$

Consequently,

$$
\begin{aligned}
& F\left(x, u_{*}(x)\right)+F^{*}\left(x, w\left((-\Delta)_{\omega}\right) u_{*}(x)\right)-\left\langle u_{*}(x), w\left((-\Delta)_{\omega}\right) u_{*}(x)\right\rangle_{\mathbb{R}}=0, \\
& \quad x \in \Omega \text { a.e. }
\end{aligned}
$$

The second part of Theorem 2 guaranties that $u_{*} \in D\left(w\left((-\Delta)_{\omega}\right)\right)$ is a solution of the inclusion (1).

The proof is completed.

Remark 5 From the above theorem it follows that if $u_{*} \in D\left(w\left((-\Delta)_{\omega}\right)\right)$ is a solution of the problem (1) then it is a minimizer of $J$. Consequently, only minimizers of $J$ can be solutions of (1) (they are in effect solutions of (1) if additionally $J\left(u_{*}\right)=0$, where $u_{*}$ denotes a minimizer of $J$ ).

Now, we formulate and prove the main result of this paper, namely a theorem on the existence of a minimizer of the functional $J$ on $D\left(w\left((-\Delta)_{\omega}\right)\right)$. We have

Theorem 4 Assume that conditions (F1)-(F3) are satisfied and

$$
c<\frac{\sqrt{M_{\beta_{k}}} \alpha_{k}}{4},
$$

where $\lambda_{1}$ is the first eigenvalue of $(-\Delta)_{\omega}$ and $c$ is a constant from the assumption (F3). Then there exists a minimizer $u_{*} \in D\left(w\left((-\Delta)_{\omega}\right)\right)$ of the functional J. Moreover, if $F(x, \cdot)$ is strictly convex on $\mathbb{R}$ for a.e. $x \in \Omega$, then the minimizer is unique.

Proof Since the space $D\left(w\left((-\Delta)_{\omega}\right)\right)$ is reflexive (as the Hilbert space), therefore it is sufficient to show that the functional $J$ is coercive and sequentially weakly lower semicontinuous on $D\left(w\left((-\Delta)_{\omega}\right)\right)$.

Coercivity: Let $\left(u_{l}\right)_{l \in \mathbb{N}} \subset D\left(w\left((-\Delta)_{\omega}\right)\right)$ be any sequence such that

$$
\left\|u_{l}\right\|_{w\left((-\Delta)_{\omega}\right)} \underset{l \rightarrow \infty}{\longrightarrow} \infty
$$


From the proof of Theorem 3 it follows that $J\left(u_{l}\right) \geq 0$. Moreover, using nonnegativity of $F$, conditions (16), (20), the Hölder inequality and the Poincaré inequality (10) we obtain

$$
\begin{aligned}
J\left(u_{l}\right) & =\int_{\Omega}\left(F\left(x, u_{l}(x)\right)+F^{*}\left(x, w\left((-\Delta)_{\omega}\right) u_{l}(x)\right)-\left\langle u_{l}(x), w\left((-\Delta)_{\omega}\right) u_{l}(x)\right\rangle_{\mathbb{R}}\right) \mathrm{d} x \\
& \geq \int_{\Omega} F^{*}\left(x, w\left((-\Delta)_{\omega}\right) u_{l}(x)\right) \mathrm{d} x-\int_{\Omega}\left\langle u_{l}(x), w\left((-\Delta)_{\omega}\right) u_{l}(x)\right\rangle_{\mathbb{R}} \mathrm{d} x \\
& \geq-\int_{\Omega} v(x) \mathrm{d} x+\frac{1}{4 c}\left\|w\left((-\Delta)_{\omega}\right) u_{l}\right\|_{L^{2}}^{2}-\left\|u_{l}\right\|_{L^{2}}\left\|w\left((-\Delta)_{\omega}\right) u_{l}\right\|_{L^{2}} \\
& \geq C+\frac{1}{4 c}\left\|u_{l}\right\|_{w\left((-\Delta)_{\omega}\right)}^{2}-\frac{1}{\sqrt{M_{\beta_{k}}} \alpha_{k}}\left\|u_{l}\right\|_{w\left((-\Delta)_{\omega}\right)}^{2} \\
& =C+\left(\frac{1}{4 c}-\frac{1}{\sqrt{M_{\beta_{k}}} \alpha_{k}}\right)\left\|u_{l}\right\|_{w\left((-\Delta)_{\omega}\right)}^{2} \underset{l \rightarrow \infty}{\longrightarrow} \infty,
\end{aligned}
$$

where $C=-\int_{\Omega} v(x) \mathrm{d} x$. This means that $J$ is coercive.

Sequential weak lower semicontinuity of $J$ : Assume that $\left(u_{l}\right)_{l \in \mathbb{N}} \subset D\left(w\left((-\Delta)_{\omega}\right)\right)$ is a sequence such that

$$
u_{l \rightarrow \infty} u_{*} \text { weakly in } D\left(w\left((-\Delta)_{\omega}\right)\right)
$$

and let

$$
J(u)=J_{1}(u)+J_{2}(u)-J_{3}(u),
$$

where

$$
\begin{aligned}
& J_{1}(u)=\int_{\Omega} F(x, u(x)) \mathrm{d} x, \quad J_{2}(u)=\int_{\Omega} F^{*}\left(x, w\left((-\Delta)_{\omega}\right) u(x)\right) \mathrm{d} x, \\
& J_{3}(u)=\int_{\Omega}\left\langle u(x), w\left((-\Delta)_{\omega}\right) u(x)\right\rangle_{\mathbb{R}} \mathrm{d} x .
\end{aligned}
$$

First, let us note that since $F$ is a Carathéodory function, therefore $\left(F\left(\cdot, u_{l}(\cdot)\right)\right)_{l \in \mathbb{N}}$ is the sequence of measurable functions. Moreover all terms of this sequence are nonnegative. Consequently, using Fatou's Lemma, we conclude

$$
\int_{\Omega} \liminf _{l \rightarrow \infty} F\left(x, u_{l}(x)\right) \mathrm{d} x \leq \liminf _{l \rightarrow \infty} \int_{\Omega} F\left(x, u_{l}(x)\right) \mathrm{d} x .
$$


[21, Proposition 3.10] implies

$$
u_{l} \underset{l \rightarrow \infty}{\longrightarrow} u_{*} \text { strongly in } L^{2}(\Omega, \mathbb{R})
$$

So, there exists a subsequent $\left(u_{l_{j}}\right)_{j \in \mathbb{N}}$ such that

$$
u_{l_{j}}(x) \underset{j \rightarrow \infty}{\longrightarrow} u_{*}(x), \quad x \in \Omega \text { a.e. }
$$

Hence and from the fact that $F$ is continuous with respect to the second variable we get

$$
\lim _{j \rightarrow \infty} F\left(x, u_{l_{j}}(x)\right)=F\left(x, u_{*}(x)\right), \quad x \in \Omega \text { a.e. }
$$

Supposing contrary and repeating the above argumentation we assert that

$$
\lim _{l \rightarrow \infty} F\left(x, u_{l}(x)\right)=F\left(x, u_{*}(x)\right), \quad x \in \Omega \text { a.e. }
$$

Consequently, the inequality (21) can be written as

$$
\int_{\Omega} F\left(x, u_{*}(x)\right) \mathrm{d} x \leq \liminf _{l \rightarrow \infty} \int_{\Omega} F\left(x, u_{l}(x)\right) \mathrm{d} x .
$$

This means that $J_{1}$ is sequentially weakly lower semicontinuous on $D\left(w\left((-\Delta)_{\omega}\right)\right)$. The functional $J_{2}$ has also such a property due to Lemma 3 .

Now, we show that $J_{3}$ is sequentially weakly continuous on $D\left(w\left((-\Delta)_{\omega}\right)\right)$. Indeed, using once again [21, Proposition 3.10] we assert that

$$
w\left((-\Delta)_{\omega}\right) u_{l} \underset{l \rightarrow \infty}{\rightarrow} w\left((-\Delta)_{\omega}\right) u_{*} \quad \text { weakly in } L^{2}(\Omega, \mathbb{R}) .
$$

This fact and convergence (22) imply

$$
\left.\lim _{l \rightarrow \infty} \int_{\Omega}\left\langle u_{l}(x), w((-\Delta))_{\omega}\right) u_{l}(x)\right\rangle_{\mathbb{R}} \mathrm{d} x=\int_{\Omega}\left\langle u_{*}(x), w\left((-\Delta)_{\omega}\right) u_{*}(x)\right\rangle_{\mathbb{R}} \mathrm{d} x
$$

Finally, we conclude that $J$ is sequentially weakly lower semicontinuous on $D\left(w\left((-\Delta)_{\omega}\right)\right)$.

Uniqueness of a solution: It is clear that since $F$ and $F^{*}$ are convex with respect to the second variable, therefore $J$ is convex on $D\left(w\left((-\Delta)_{\omega}\right)\right)$. Let us suppose that $u_{*}$ and $v_{*}$ are two different minimizers of $J$ on $D\left(w\left((-\Delta)_{\omega}\right)\right)$ and assume that $F(x, \cdot)$ is strictly convex on $\mathbb{R}$ for a.e. $x \in \Omega$. This implies that $J_{1}$, so also $J$, are strictly convex on $D\left(w\left((-\Delta)_{\omega}\right)\right)$. This means in particular that

$$
J\left(\frac{u_{*}+v_{*}}{2}\right)<J\left(u_{*}\right)=J\left(v_{*}\right) .
$$


This contradicts the assumption that $u_{*}\left(v_{*}\right)$ is a minimizer of $J$ on $D\left(w\left((-\Delta)_{\omega}\right)\right)$. The proof is completed.

Remark 6 Let us note that only the order $\beta_{k}$ and the value $\alpha_{k}$ impact the existence of a minimizer of $J$. This is a consequence of the fact that domains $D\left(\left((-\Delta)_{\omega}\right)^{\beta_{k}}\right)$ and $D\left(w\left((-\Delta)_{\omega}\right)\right)$ coincide.

\section{Illustrative Examples}

In this section we present two simple theoretical examples which illustrate results obtained in Sect. 3.

Example 1 Let us consider the following problem:

$$
\left((-\Delta)_{\omega}\right)^{\frac{1}{3}} u(x)+5\left((-\Delta)_{\omega}\right)^{\frac{1}{2}} u(x) \in \partial_{u} F(x, u(x)), \quad x=\left(x_{1}, x_{2}\right) \in \Omega \text { a.e. }
$$

where $\Omega=(0, \pi) \times(0, \pi) \subset \mathbb{R}^{2}$ and $F: \Omega \times \mathbb{R} \rightarrow[0, \infty)$ is given by

$$
F(x, u):= \begin{cases}|u| & \text { if }|u| \leq 1 \\ u^{2} & \text { if }|u|>1\end{cases}
$$

It is easy to check that

$$
\partial_{u} F(x, u)= \begin{cases}{[-2,-1]} & \text { if } u=-1 \\ \{-1\} & \text { if } u \in(-1,0) \\ {[-1,1]} & \text { if } u=0 \\ \{1\} & \text { if } u \in(0,1) \\ {[1,2]} & \text { if } u=1 \\ \{2 u\} & \text { if }|u|>1 .\end{cases}
$$

Moreover,

$$
\begin{aligned}
F^{*}(x, z) & =\sup _{u \in \mathbb{R}}\left[\langle u, z\rangle_{\mathbb{R}}-F(x, u)\right] \\
& =\max \left\{\sup _{|u| \leq 1}\left[\langle u, z\rangle_{\mathbb{R}}-|u|\right], \sup _{|u|>1}\left[\langle u, z\rangle_{\mathbb{R}}-u^{2}\right]\right\} .
\end{aligned}
$$


We check that

$$
\sup _{|u| \leq 1}\left[\langle u, z\rangle_{\mathbb{R}}-|u|\right]= \begin{cases}-z-1 & \text { if } z<-1 \\ z-1 & \text { if } z>1 \\ 0 & \text { if }|z| \leq 1\end{cases}
$$

and

$$
\sup _{|u|>1}\left[\langle u, z\rangle_{\mathbb{R}}-u^{2}\right]= \begin{cases}-z-1 & \text { if } z \in[-2,0] \\ z-1 & \text { if } z \in[0,2] \\ \frac{1}{4} z^{4} & \text { if }|z|>2\end{cases}
$$

Consequently,

$$
F^{*}(x, z)= \begin{cases}0 & \text { if }|z|<1 \\ -z-1 & \text { if } z \in[-2,-1] \\ z-1 & \text { if } z \in[1,2] \\ \frac{1}{4} z^{4} & \text { if }|z|>2 .\end{cases}
$$

Of course assumptions (F1)-(F3) are satisfied. In particular,

$$
F(x, u) \leq 1+|u|^{2},
$$

so the condition (13) holds with $c=1$. Moreover, it is well known (cf. [2, Proposition 8.5.3]) that the first eigenvalue of $(-\Delta)_{\omega}$ equals to 2 , so the assumption (20) is also satisfied. Consequently, using Theorem 4, we assert that there exists a minimizer (not necessarily unique) of the functional $J$ given by (17) which can be the only possible solution of the problem (23) (cf. Remark 5).

Example 2 Let us consider the following boundary value problem:

$$
\sum_{i=0}^{k} \alpha_{i}\left((-\Delta)_{\omega}\right)^{\beta_{i}} u(x)=D_{u} F(x, u(x)), \quad x \in \Omega \text { a.e. }
$$

where $\alpha_{i}>0, i=0, \ldots, k(k \in \mathbb{N} \cup\{0\}), 0 \leq \beta_{0}<\beta_{1}<\cdots<\beta_{k}, \Omega \subset \mathbb{R}^{N}$, is any open and bounded set, $F: \Omega \times \mathbb{R} \rightarrow[0, \infty)$ is given by

$$
F(x, u):=\frac{a(x)}{p}|u|^{p}
$$

where $1<p<2$ and $a \in L^{\infty}\left(\Omega, \mathbb{R}_{+}\right)$. 
We shall show that the above problem has the only trivial solution. Indeed, first let us note that

$$
F^{*}(x, z)=\frac{1}{q a(x)}|z|^{q}, \quad \frac{1}{p}+\frac{1}{q}=1
$$

and the partial derivative of $F$ with respect to $u$ is given by

$$
D_{u} F(x, u)=a(x)|u|^{p-2} u .
$$

Moreover, assumptions (F1)-(F3) hold. In particular, for any $c>0$ there exists a sufficiently large constant $R>0$ (dependent on the constants $c, p$ and the function $a$ ) such that

$$
\frac{a(x)}{p}|u|^{p} \leq \frac{\|a\|_{L^{\infty}}}{p}|u|^{p} \leq \begin{cases}c|u|^{2}, & |u|>R \\ \frac{\|a\|_{L^{\infty}}}{p} R^{p}+c|u|^{2}, & |u| \leq R\end{cases}
$$

so, the condition (13) is satisfied for any $c>0$.

Consequently, since all assumptions of Theorem 4 are satisfied and $F$ is strictly convex with respect to $u$ for a.e. $x \in \Omega$, therefore the functional $J$ given by

$$
J(u)=\int_{\Omega}\left(\frac{a(x)}{p}|u(x)|^{p}+\frac{1}{q a(x)}\left|\left[w\left((-\Delta)_{\omega}\right) u(x)\right]\right|^{q}-u(x) w\left((-\Delta)_{\omega}\right) u(x)\right) \mathrm{d} x
$$

has a unique minimizer $u_{*} \in D\left(w\left((-\Delta)_{\omega}\right)\right)$.

On the other hand it is clear that $u_{*}=0$ is a solution of the problem (24), so it must minimize $J$ on $D\left(w\left((-\Delta)_{\omega}\right)\right)$. This means that $u_{*}=0$ is the only solution of (24).

Remark 7 If $p=2$ in the above example then the condition (13) holds for $c=\frac{\|a\|_{L^{\infty}}}{2}$. Consequently, the linear problem of the form

$$
\sum_{i=0}^{k} \alpha_{i}\left((-\Delta)_{\omega}\right)^{\beta_{i}} u(x)=a(x) u(x), \quad x \in \Omega \text { a.e. }
$$

has the only trivial solution provided that

$$
\|a\|_{L^{\infty}}<\frac{\sqrt{M_{\beta_{k}}} \alpha_{k}}{2}
$$


Here it is worth to note that replacing the functional $J$ given by (25) (for $p=q=2$ ) with the following one

$$
\begin{aligned}
J_{1}(u)= & \int_{\Omega}\left(\frac{a(x)}{2}|u(x)|^{2}+\frac{1}{2 a(x)}\left|\left[w\left((-\Delta)_{\omega}\right) u(x)-f(x)\right]\right|^{2}\right. \\
& \left.-u(x)\left(w\left((-\Delta)_{\omega}\right) u(x)-f(x)\right)\right) \mathrm{d} x
\end{aligned}
$$

we can prove (similarly as in Sect. 3) counterparts of Theorems 3 and 4 for the functional $J_{1}$ (in particular, under assumption (26) one can obtain the existence of a unique minimizer of $J_{1}$ on $\left.D\left(w\left((-\Delta)_{\omega}\right)\right)\right)$ and the following, more general linear problem

$$
\sum_{i=0}^{k} \alpha_{i}\left((-\Delta)_{\omega}\right)^{\beta_{i}} u(x)=a(x) u(x)+f(x), \quad x \in \Omega \text { a.e. }
$$

where $f \in L^{2}(\Omega, \mathbb{R})$. Additionally, if $a$ is a constant function, then the problem (27) has a unique solution. Indeed, since $J_{1}$ has a unique minimizer $u_{*}$ on $D\left(w\left((-\Delta)_{\omega}\right)\right)$, therefore

$$
\delta J_{1}\left(u_{*}, h\right)=0 . \quad h \in D\left(w\left((-\Delta)_{\omega}\right)\right)
$$

where $\delta J_{1}\left(u_{*}, h\right)$ denotes the first variation of $J_{1}$ at $u_{*}$ in the direction $h$.

So,

$$
\begin{gathered}
\delta J_{1}\left(u_{*}, h\right)=\int_{\Omega} \frac{1}{a}\left(w\left((-\Delta)_{\omega}\right) u_{*}(x)-a u_{*}(x)-f(x)\right) \\
\left(w\left((-\Delta)_{\omega}\right) h(x)-a h(x)\right) \mathrm{d} x=0
\end{gathered}
$$

for any $h \in D\left(w\left((-\Delta)_{\omega}\right)\right)$.

Since $a<\frac{\sqrt{M_{\beta_{k}}} \alpha_{k}}{2}$, therefore the constant $a$ is not the eigenvalue of the operator $w\left((-\Delta)_{\omega}\right)$. Consequently, the kernel of the self-adjoint operator $L: D\left(w\left((-\Delta)_{\omega}\right)\right) \rightarrow$ $L^{2}(\Omega, \mathbb{R})$, defined as

$$
L h:=w\left((-\Delta)_{\omega}\right) h-a h, \quad a>0,
$$

is trivial, so the image of $L$ satisfies the equality $R(L)=L^{2}(\Omega, \mathbb{R})$. Hence and from (29) we conclude

$$
w\left((-\Delta)_{\omega}\right) u_{*}(x)-a u_{*}(x)-f(x)=0, \quad x \in \Omega \text { a.e. }
$$

This means that $u_{*}$ is a unique solution of (27). 
Open Access This article is licensed under a Creative Commons Attribution 4.0 International License, which permits use, sharing, adaptation, distribution and reproduction in any medium or format, as long as you give appropriate credit to the original author(s) and the source, provide a link to the Creative Commons licence, and indicate if changes were made. The images or other third party material in this article are included in the article's Creative Commons licence, unless indicated otherwise in a credit line to the material. If material is not included in the article's Creative Commons licence and your intended use is not permitted by statutory regulation or exceeds the permitted use, you will need to obtain permission directly from the copyright holder. To view a copy of this licence, visit http://creativecommons.org/licenses/by/4.0/.

\section{References}

1. Applebaum, D.: Lévy processes-from probability to finance and quantum groups. Notices Am. Math. Soc. 51, 1336-1347 (2004)

2. Attouch, H., Buttazzo, G., Michaille, G.: Variational Analysis in Sobolev and BV Spaces. Applications to PDEs and Optimization. SIAM-MPS, Philadelphia (2006)

3. Auchmuty, G.: Variational principles for finite dimensional initial value problems. Contemp. Math. 426, 45-56 (2007)

4. Barrios, B., Colorado, E., de Pablo, A., Sánchez, U.: On some critical problems for the fractional Laplacian operator. J. Differ. Equ. 252, 6133-6162 (2012)

5. Bermudez, A., Saguez, C.: Optimal control of a Signorini problem. SIAM J. Control Optim. 25, 576-582 (1987)

6. Bogdan, K., Byczkowski, T.: Potential theory of Schrdinger operator based on fractional Laplacian. Probab. Math. Stat. 20(2), 293-335 (2000)

7. Bogdan, K., Byczkowski, T., Kulczycki, T., Ryznar, M., Song, R., Vondracek, Z.: Potential Theory of Stable Processes and its Extensions. Lecture Notes in Mathematics, vol. 1980. Springer, Berlin (2009)

8. Bonforte, M., Vázquez, J.L.: A priori estimates for fractional nonlinear degenerate diffusion equations on bounded domains. The Royal Swedish Academy of Sciences, Mittag-Leffler Institute Report No. 21, 2013/2014. arXiv:1311.6997

9. Bors, D.: Optimal control of nonlinear systems governed by Dirichlet fractional Laplacian in the minimax framework (2017). arXiv:1509.01283

10. Cabré, X., Tan, J.: Positive solutions of nonlinear problems involving the square root of the Laplacian. Adv. Math. 224, 2052-2093 (2010)

11. Caffarelli, L.A., Salsa, S., Silvestre, L.: Regularity estimates for the solution and the free boundary of the obstacle problem for the fractional Laplacian. Invent. Math. 171, 425-461 (2008)

12. Caffarelli, L.A., Vasseur, A.: Drift diffusion equations with fractional diffusion and the quasigeostrophic equation. Ann. Math. 171, 1903-1930 (2010)

13. Chen, Z.-Q., Song, R.: Two-sided eigenvalue estimates for subordinate Brownian motion in bounded domains. J. Funct. Anal. 226, 90-113 (2005)

14. Cont, R., Tankov, P.: Financial Modelling with Jump Processes. Chapman \& Hall/CRC Financial Mathematics Series. Chapman \& Hall/CRC, Boca Raton (2004)

15. Di Nezza, E., Palatucci, G., Valdinoci, E.: Hitchhiker's guide to the fractional Sobolev spaces. Bull. Sci. Math. 136(5), 521-573 (2012)

16. Fitzpatrick, P.M.: A note on the functional calculus for unbounded self-adjoint operators. J. Fixed Point Theory Appl. 13, 633-640 (2013)

17. Grubb, G.: Fractional Laplacians on domains, a development of Hrmanders theory of $\mu$-transmission pseudodifferential operators. Adv. Math. 268, 478-528 (2015)

18. Grubb, G.: Regularity of spectral fractional Dirichlet and Neumann problems. Math. Nachr. 289(7), 831-844 (2016)

19. Hiriart-Urruty, J.M., Lemarchal, C.: Fundamentals of Convex Analysis. Springer, Berlin (2001)

20. Idczak D.: Maximum principle for fractional ordinary Dirichlet-Laplace problem with integral performance index via Dubovitskii-Miljutin method, accepted for publication in Nonlinear Analysis: Modelling and Control

21. Idczak, D.: A bipolynomial fractional Dirichlet-Laplace problem. Electron. J. Differ. Equ. 2019(59), 1-17 (2019)

22. Jost, J., Li-Jost, X.: Calculus of Variations. Cambridge University Press, Cambridge (1998) 
23. Kowalski, E.: Spectral Theory in Hilbert Spaces (ETH Zurich, FS 09)

24. Landkof, N.: Foundations of Modern Potential Theory. Springer, New York (1972). (Translated from Russian by Doohovskoy AP)

25. Reed, M., Simon, B.: Methods of Modern Mathematical Physics. Academic Press, Cambridge (1980)

26. Rockafellar, R.T.: Convex Analysis. Princeton University Press, New Jersey (1970)

27. Schilling, R.L., Song, R., Vondracek, Z.: Bernstein Functions: Theory and Applications, volume 37 of de Gruyter Studies in Mathematics, 2nd edn. Walter de Gruyter \& Co., Berlin (2012)

28. Tan, J.: The Brezis-Nirenberg type problem involving the square root of the Laplacian. Calc. Var. 42, 21-41 (2011)

29. Vázquez, J.L.: Nonlinear diffusion with fractional Laplacian operators. In: Nonlinear Partial Differential Equations, vol. 7 of Abel Symposia, pp. 271-298 (2012)

30. Vázquez, J.L.: Recent progress in the theory of nonlinear diffusion with fractional Laplacian operators. Discrete Contin. Dyn. Syst. Ser. S 7, 857-885 (2014)

Publisher's Note Springer Nature remains neutral with regard to jurisdictional claims in published maps and institutional affiliations. 